\title{
ILCEA
}

Revue de l'Institut des langues et cultures

d'Europe, Amérique, Afrique, Asie et Australie

13 | 2010

Les voies incertaines de la démocratisation

\section{Révolution des CEillets : transition sociopolitique et démocratisation au Portugal}

Ana Saldanha

\section{OpenEdition}

Journals

Édition électronique

URL : http://journals.openedition.org/ilcea/872

DOI : 10.4000/ilcea.872

ISSN : 2101-0609

\section{Éditeur}

UGA Éditions/Université Grenoble Alpes

Édition imprimée

ISBN : 978-2-84310-181-6

ISSN : $1639-6073$

Référence électronique

Ana Saldanha, « Révolution des Fillets : transition sociopolitique et démocratisation au Portugal », ILCEA [En ligne], 13 | 2010, mis en ligne le 30 novembre 2010, consulté le 21 décembre 2020. URL: http://journals.openedition.org/ilcea/872 ; DOI : https://doi.org/10.4000/ilcea.872

Ce document a été généré automatiquement le 21 décembre 2020.

(C) ILCEA 


\title{
Révolution des đillets : transition sociopolitique et démocratisation au Portugal
}

\author{
Ana Saldanha
}

1 L'ouverture d'une nouvelle époque en opposition à une autre, répressive et autoritaire, est aujourd'hui appelée transition démocratique et est assimilée à un certain type de modèle de changement politique: la transition démocratique est ainsi une catégorie historique et politique qui existe en parallèle à celles de révolution, évolution, rupture, Or, si l'on entend transition politique en un sens strict, elle représente donc un changement politique contrôlé qui s'opère au sein de certaines sociétés et qui permet le passage d'un système à un autre sans que l'on puisse délimiter le moment précis de rupture. En ce sens, transition, rupture et révolution constituent des catégories différentes, la première représentant, par exemple, le cas transitionnel de l'Espagne, après la mort de Franco, en 1975.

Nous allons cependant considérer la transition comme un processus de changement qui peut être effectué à travers une rupture politique et économique avec un régime antérieur. Sur ce point, nous suivons donc la catégorisation proposée par Munck et Leff (1997), selon laquelle il existe une transition qui coupe radicalement avec le passé, en permettant la rénovation des élites et l'apparition d'un nouveau cadre institutionnel. Cependant, nous constatons que l'action des non-élites a été cruciale pour que le processus de transition par rupture puisse s'effectuer. En ce sens, nous n'attribuons pas le rôle primordial de la transition au mode de transition et au conflit qui en découle entre les élites et les counterelites ${ }^{1}$ (que nous considérons être le mouvement des capitaines). Nous considérons que le rôle des mouvements sociaux et/ou populaires et politiques (les organisations des étudiants, des travailleurs des paysans, des femmes, des communistes, des antifascistes et d'autres démocrates, c'est-à-dire, des non-élites) a été fondamental dans la rupture causée par la chute du régime, ceci sans ignorer le rôle qu'a eu la crise économique de 1973 (Muñoz, 1997). 
3 En 1973, un groupe d'officiers de carrière entame un mouvement corporatiste qui, peu à peu, s'amplifie, transformant les revendications initiales en une volonté de changement de régime. C'est ce mouvement qui conduira, le 25 avril 1974, à la chute de la dictature la plus longue de l'Europe. Les manifestations populaires de soutien qui suivent, constituent, de leur côté, la base du double caractère de cette révolution: étant initialement une révolution militaire, elle se transforme en une révolution militaire et populaire. C'est donc ce double caractère qui permet la coupure avec l'ancien mode d'organisation sociopolitique et économique ainsi que l'institutionnalisation d'un nouvel ordre.

Dans cet article nous chercherons à analyser, dans un premier temps, les raisons qui ont conduit la chute du régime dictatorial portugais et, dans un deuxième temps, le rôle de chacun des principaux protagonistes de la révolution et du processus de transition démocratique pour, finalement, comprendre la spécificité du processus révolutionnaire portugais ainsi que de la transition de la dictature à un nouvel ordre sociopolitique et économique. Cette transition initie, selon Huntington (1993), la «troisième vague de démocratisation ${ }^{2}$ ", dans laquelle s'insère, également, la chute du régime militaire grec (trois mois après la révolution des (Eillets) et le début de la transition espagnole, après la mort de Franco, en 1975.

\section{De la dictature militaire à l'instauration de l'autoproclamé « État Nouveau »}

5 La République portugaise instaurée en 1910 a été très instable, présentant des contradictions internes et des problèmes structuraux que les républicains n'ont pas su solutionner. Parallèlement, les constantes attaques des monarchistes, la crise économique (nationale et européenne) et la constante agitation sociale ont aggravé le mécontentement social, surtout au sein des classes travailleuses les plus dépourvues. De son côté, la grande industrie et la banque cherchaient à instaurer un climat de tranquillité sociale pour le développement de ses ambitions croissantes, tandis que l'Église catholique cherchait à récupérer les privilèges perdus lors de l'institutionnalisation de la République laïque. Dans ce contexte, les hiérarchies de l'armée jugeaient nécessaire leur intervention pour le rétablissement de l'ordre et la fin de l'agitation sociale.

6 C'est ainsi que le 28 mai 1926 un coup d'État venant des filières de la droite militaire a eu lieu, imposant une dictature qui ne finira que 48 ans plus tard. La première République prit fin et les progrès sociaux et politiques entamés lors des 16 dernières années sont remplacés par une politique de régression, de répression et de connivence avec l'Église catholique. L'on est, selon Huntington (1993), dans la « première vague inverse ${ }^{3}$ » qui suit l'implantation de la "première vague de démocratisation » entre 1828 et 1926 (et initiée par les États-Unis d'Amérique) ${ }^{4}$.

7 Jusqu'en 1933, le Portugal a été gouverné par une dictature militaire typique, pendant laquelle ont été supprimés les libertés et les droits fondamentaux mais « sans procéder à l'institutionnalisation d'une nouvelle structure de l'État » (Cunhal, 1999, p. 27). Entre 1928 et 1930 un professeur d'économie de l'université de Coimbra, António de Oliveira Salazar (1889-1970), a été nommé ministre des Finances. Face à la catastrophique situation économique du Portugal, Salazar a redressé les finances (même si les méthodes utilisées 
pour justifier ce redressement sont contestées par certains économistes), ce qui lui a permis d'apparaître face à la nation comme le "sauveur» des finances publiques et comme celui qui a restitué un ordre perdu. Ces résultats ont également permis à Salazar de s'immiscer peu à peu dans les problèmes politiques et militaires : il avait l'appui de la banque, du capital (tous les deux désiraient s'épandre sans restrictions ainsi que se protéger des luttes de classe, des mouvements de grève et de l'agitation sociale), de l'Église, d'intellectuels de droite, pour la plupart des professeurs de l'université de Coimbra, ainsi que des monarchistes, convaincus que Salazar réinstaurerait la monarchie. En 1932, Salazar a été nommé président du Conseil (actuellement, Premier ministre).

8 La période qui s'initie avec Oliveira Salazar comme président du Conseil correspond à la création de l'État corporatif et à l'institutionnalisation de la dictature fasciste (Cunhal, 1999, p. 27), avec l'approbation, en 1933, d'une nouvelle Constitution. Cette Constitution établit les principes constitutionnels qui ont permis l'activité totalitaire et répressive de l'État (qui s'est auto-dénommé État Nouveau) : elle a instauré la censure, a interdit les partis politiques, les Associations syndicales et les Associations secrètes, a créé la PVDE ${ }^{5}$ - police politique -, a interdit l'opposition et a imposé le parti unique.

9 Sur le plan économique, la dictature procéda à l'accumulation capitaliste: de grands monopoles surgirent alors au Portugal dont la dimension et le pouvoir économique se sont révélés être démesurés dans un pays sous-développé qui était l'un des plus pauvres de l'Europe (Oliveira Marques, 2001). La dictature portugaise a toutefois été tolérée par les régimes européens. La Grande-Bretagne, traditionnelle alliée économique du Portugal depuis le XIV siècle, s'est tranquillisée avec la fin de la période d'agitation sociale qui avait menacé ses intérêts économiques au Portugal. La France, oubliant la pression exercée par les exilés politiques portugais dans son pays, a recommencé ses relations avec le Portugal, tandis que la dictature espagnole de Primo de Rivera a applaudi le nouvel ordre du pays voisin. La proclamation de la République espagnole, en 1931, a inquiété le gouvernement portugais, cependant son virement vers la droite a fait croire à Salazar que les relations entre les deux pays allaient rester proches. Quand le Front populaire a été élu en 1936, Salazar, avec Mussolini et Hitler, a soutenu Franco et l'armée contrerévolutionnaire, fournissant des armes, des aliments et des munitions, cela malgré l'accord de non-intervention qui avait été signé avec la France et la Grande-Bretagne.

10 La déroute des fascismes en 1945 correspond, selon la catégorisation de Huntington (1993), à la "deuxième vague de démocratisation ${ }^{6}$ " (Allemagne occidentale, Japon, Autriche, Italie et Corée). Cela ne signifie pas pour autant la fin des régimes dictatoriaux en Espagne ou au Portugal. Les régimes occidentaux, craignant l'avancée communiste et la possibilité d'un contrôle de la péninsule Ibérique par les partis communistes, ont soutenu Salazar, au Portugal, et Franco, en Espagne. Le Portugal a été membre fondateur de l'OTAN et, en 1955, est entré à l'ONU : à l'inverse de ce que l'on pourrait croire, la répression augmenta d'intensité.

11 À côté des mouvements sociaux et politiques qui combattaient (clandestinement) le régime, un nouvel acteur va prendre une place importante dans la scène politique nationale portugaise : les militaires. Ainsi, à la fin des années 1950, début des années 1960, une opposition s'est manifestée à l'intérieur des forces armées, annonçant un mal-être qui laissait présager de la formation, plus de dix ans plus tard, du Mouvement des Forces armées. Le soutien des forces armées à la dictature s'est détérioré encore plus avec l'avènement de la guerre coloniale. 


\section{Le début de la guerre coloniale et le mûrissement des conditions pour la Révolution}

12 La fin de la Seconde Guerre mondiale a permis le « réveil du continent africain » (Oliveira Marques, 2001, p. 701) au sein duquel se sont alors développés des mouvements de libération. Les colonies portugaises ont suivi cette tendance et divers groupes d'unité africaine ont été constitués.

13 Ces mouvements de libération ont été formés, en général, par une bourgeoisie créole qui a pu suivre ses études en métropole. En 1956, sont créés le MPLA (Angola) et le PAIGCV ${ }^{8}$ (Guinée et Cap-Vert), en 1961 est créé le MLSTP ${ }^{9}$ (Sao Tomé-et-Principe) et en 1962 le FRELIMO $^{10}$ (Mozambique). Néanmoins, le FRELIMO, à l'inverse des mouvements angolais, guinéens et santoméens, a été fondé par des Africains qui n'avaient pas toujours de liaison avec la culture et la langue portugaise. Ces quatre mouvements ont légitimé leur action selon les préceptes marxistes.

14 Le gouvernement de Salazar a répondu aux mouvements indépendantistes par la répression et la guerre. Celle-ci a commencé en février 1961, en Angola, et s'est étendue, en 1963, à la Guinée-Bissau et, en 1964, au Mozambique. Au début des années 1960, le mouvement anticolonialiste dans d'autres pays africains se trouvait toutefois déjà assez développé. Cet avancementdes mouvements indépendantistes a donc obligé Salazar à recourir à une alliance avec les États colonialistes, en particulier l'Afrique du Sud, la Rhodésie et le Nyassaland (nom colonial du Malawi jusqu'en 1964). À la fin de l'année 1963, Salazar a écrit au Premier ministre de l'Afrique du Sud, H. Verwoerd :

La lutte subversive [...] est très loin de vos frontières, mais elle arrivera rapidement à vous si le Portugal n'arrive pas à résister. Cela veut dire qu'il y a un intérêt occidental et surtout de l'Afrique du Sud d'empêcher une telle situation, et pour cela toutes formes de coopération avec le Portugal dans les territoires d'Angola et du Mozambique doivent être envisagées, pour votre propre défense. (Ramos de Almeida, 2002, p. 40-41.)

À l'inverse des attentes du régime, le début de la guerre coloniale a permis le développement des actions et des théories anticolonialistes et l'opposition à la guerre va s'étendre à différents secteurs de la population. En 1961, huit prêtres angolais ont été déportés vers le Portugal, emprisonnés et torturés; en 1965 a été divulgué le «Manifeste des 101 catholiques qui assumait ouvertement une position contre la guerre coloniale, critiquant la complicité de la hiérarchie catholique avec le régime de Salazar " (Ramos de Almeida, 2002, p. 45). Le traditionnel appui de l'Église catholique au régime était alors remis en cause.

De son côté, le Parti communiste portugais (PCP), le seul parti organisé clandestinement dès le début de la dictature ${ }^{11}$, a appelé à la désertion des jeunes des forces armées. Cet appel et l'incompréhension d'une guerre qui ne cessait d'augmenter l'envoi de jeunes incorporés en Afrique a conduit beaucoup d'entre eux à choisir la désertion : le chiffre des désertions a atteint $11,68 \%$ en $1961,12,8 \%$ en $1962,18,6 \%$ en 1966 , tandis que pendant les années 1970, 1971 et 1972 ce chiffre est monté à plus de $20 \%$ (Ramos de Almeida, 2002).

17 À la fin de l'année 1961, les troupes de Nehru ont envahi les colonies portugaises en Inde (Goa, Damão et Diu) : le gouverneur général de l'État portugais des Indes a contredit les ordres donnés par Salazar de "résister jusqu'à la mort » aux troupes indiennes et a 
délivré, sans résistance, les colonies à l'état indien. C'était le début de la fin de l'empire colonial tant exalté par le fascisme portugais.

Or, les forces armées ont été la base d'appui de la dictature tout au long de son existence. Il fallait donc que la rupture parte de ces mêmes forces qui jadis avaient permis sa survivance.

\section{La crise économique et sociale, les luttes sociales et les divisons au sein de la dictature}

Rafael Durán Muñoz (1997) défend la prépondérance du facteur économique pour justifier la chute du régime dictatorial portugais et la transition démocratique ${ }^{12}$. En ce sens, la crise économique de 1973 est vue comme un élément qui a forcé ou a facilité les démocratisations de la péninsule Ibérique.

Certes, l'économie portugaise (ainsi qu'espagnole) a été atteinte par la crise internationale de l'énergie en 1973 car « toutes les deux étaient très sensibles à la grande situation de dépendance au tourisme, aux envois d'argent des travailleurs émigrants, à l'investissement étranger [...] et au commerce externe» (Muñoz, 1997, p. 381). Il faut toutefois rajouter à cette crise la centralisation, la concentration et l'accumulation du capital des grands groupes économiques qui, dans une « économie en retard comme celle du Portugal ont créé de nouvelles contradictions et problèmes, ainsi que la détérioration de la situation économique et sociale» (Cunhal, 1999, p. 84). Cette situation a conséquemment créé une croissante montée des prix (11,5\% en 1972 et 19,2\% en 1973), provoquant la baisse des salaires réels, la baisse du pouvoir d'achat et l'augmentation du chômage (Cunhal, 1999).

21 Les mouvements d'étudiants et des travailleurs ont répondu à la crise par des grèves et des manifestations, augmentant la tension sociale et contribuant à l'accentuation de la crise générale de la dictature. La détérioration de la situation économique et sociale et l'incapacité du gouvernement d'y faire face a aussi été suivie par des divisions et des conflits au sein du pouvoir et, par conséquent, par l'isolement croissant de la dictature, au niveau interne ainsi qu'externe. Au sein même de la seule structure politique autorisée - l'Union nationale (renommée Action nationale populaire [ANP] par Marcello Caetano) les divergences et confrontations ont eu lieu. Les ultras réclamaient un durcissement du système autoritaire et l'anéantissement de l'opposition tandis que les plus libéraux réclamaient plus d'ouverture. Les confrontations entre ces deux branches du régime ont eu comme conséquence la réduction de sa base de soutien, provoquant le passage à l'opposition de quelques cadres du régime.

Or, l'opposition des cadres du régime, de l'Armée et de l'Église ainsi que l'augmentation de la lutte des ouvriers industriels, des ouvriers agricoles, des pêcheurs, des travailleurs du tertiaire, des étudiants, des communistes et des antifascistes en général a créé une situation de rupture qui ne pouvait qu'amener à une situation révolutionnaire. Aux différents mouvements sociaux et de classe qui proliféraient, le gouvernement répondait par la violence, la fermeture d'organisations professionnelles et de classes, l'emprisonnement et la torture ou l'incorporation des opposants dans les forces armées.

Ne pas avoir en considération l'action développée par les mouvements sociaux et politiques tout au long du régime de Salazar (puis, depuis 1969, de Marcello Caetano), c'est nier un aspect central qui a conduit à la désagrégation de la dictature et qui nous 
permet également de mieux comprendre le caractère populaire de la révolution portugaise ainsi que les choix politiques qui ont alors été faits.

\section{La Révolution du 25 avril 1974} Marcello Caetano le 25 avril 1974 n'est pas unanime : l'on parle de coup d'État militaire, de processus révolutionnaire, de révolte. Or, nous considérons que dans la nuit du 24 au 25 avril, il y a eu un coup d'État militaire qui prétendait prendre le pouvoir mais qui s'est transformé, très rapidement, en une révolution. Ainsi, le soulèvement militaire a été suivi $1^{\circ}$ d'un soulèvement dans d'autres unités militaires qui ne participaient pas au mouvement initial des capitaines et $2^{\circ}$ d'une forte mobilisation et intervention populaires. La synthèse qui en résulte a ainsi transformé ce qui semblait être un simple coup d'État militaire en une révolution. Cette union a d'ailleurs constitué la particularité du processus révolutionnaire portugais et marque les transformations sociales qui suivront. De cette union est née la consigne Peuple-MFA, qui symbolise l'étroite relation qui s'est établie entre les forces armées et la population.

Dans la Radio Clube Português, le 25 avril 1974 à 4 heures, le premier communiqué du Mouvement des Forces armées (MFA) ${ }^{13}$ a été lu: "Les Forces armées portugaises appellent la population de Lisbonne à rester chez soi dans le plus grand calme. » Les consignes des capitaines n'ont pas été suivies et la population de Lisbonne est immédiatement sortie dans les rues : c'est le début du soulèvement populaire.

Le MFA était pourtant un mouvement assez hétérogène. Dès le début les différences au sein de sa structure se sont fait sentir et ce particulièrement lors de la formation des organes provisoires du pouvoir ${ }^{14}$. L'organe provisoire constitué pour gouverner, la Junta de Salvação Nacional ${ }^{15}$ (JSN), était présidé par António de Spínola, un général qui avait des différends avec l'ancien régimemais qui ne prétendait pas détruire les bases du système dictatorial, défendant la continuation de la PIDE-DGS ${ }^{16}$, l'interdiction du Parti communiste portugais (PCP), le maintien en prison de certains prisonniers politiques et proposant une solution fédéraliste pour les colonies. Outre son président, la JSN incluait en son sein des généraux et autres membres de la hiérarchie militaire qui venaient de l' ancien régime. C'est ainsi que depuis la reddition du président du Conseil, Marcello Caetano, le 25 avril 1974, Spínola et d'autres anciennes élites ont insisté sur la nécessité de réviser le programme du MFA, prétendant une réforme du régime sans révolutionner sa base antidémocratique.

La JSN était donc composée d'éléments qui ne partageaient ni les objectifs définis dans le programme du MFA ni les propositions qui y étaient faites. Désormais, António de Spínola a été nommé président de la République (en attendant l'organisation des élections) le 15 mai 1974.

28 D'un autre côté, ceux qui détenaient le pouvoir économique ont été dès lors inquiets pour la sauvegarde de leurs intérêts et possessions: il y a eu des transferts illégaux de capitaux, des annulations de commandes, des paralysies de la production, des transferts illégaux d'animaux vers l'Espagne et des champs de céréales brûlés. Ces tentatives de déstabilisation économique ont eu comme réponse l'occupation d'usines et de terres, les travailleurs prenant en main la production et l'exploitation industrielle et agricole. Ainsi sont nées des organisations populaires autonomes : des commissions de travailleurs, de

ILCEA, 13 | 2010 
résidents de quartier, de contrôle et de gestion d'entreprises (qui ont été abandonnées par leurs propriétaires), d'occupation et d'exploitation de terres (pour la plupart incultes ou abandonnées). Ces organisations (impulsées par les non-élites) ont été la base d'une nouvelle forme d'organisation sociale et économique et ont eu un rôle fondamental dans le processus de rupture révolutionnaire avec l'ancien régime.

\section{L'évolution du processus révolutionnaire et la contre- révolution du 25 novembre 1975}

Le 16 mai 1974 a été formé le premier gouvernement provisoire, avec la participation de toutes les tendances politiques. Dès lors ont commencé les tentatives d'António de Spínola pour renverser politiquement et militairement le nouvel ordre révolutionnaire. Le 13 juin, lors d'une réunion entre la JSN et le MFA, Spínola a demandé les pleins pouvoirs et a attaqué la Commission coordinatrice du MFA (organisme politique et militaire du MFA), faisant appel à la discipline et à l'obéissance, contre la remise en cause des hiérarchies au sein des Forces armées (FA). Les 8 et 10 juillet, le Premier ministre, Palma Carlos (en connivence avec Spínola), a présenté, respectivement, au Conseil d'État et au Conseil des ministres un Projet de Loi Constitutionnel dans lequel il proposait la dissolution de la JSN et du MFA et l'attribution au président de la République du pouvoir suprême sur les FA. Les deux Conseils ont refusé cette proposition et trois ministres ont alors démissionné du gouvernement. Le 18 juillet, un $2^{\mathrm{e}}$ gouvernement provisoire a été mis en place ayant comme Premier ministre le lieutenant-colonel Vasco Gonçalves, de la gauche militaire et proche des positions et propositions politiques présentées par le Parti communiste portugais (PCP).

Spínola a continué, sans pourtant y réussir, ses tentatives de dissolution du MFA.

Le 28 septembre 1974 il a appelé à une grande mobilisation nationale, la majorité silencieuse, qui devait se concentrer à Lisbonne, devant l'Assemblée constituante, et lui demander de sauver le pays du chaos, du communisme et du gouvernement de Vasco Gonçalves. Cette manifestation avait l'appui des élites militaires, économiques et politiques de l'ancien régime, ainsi que le soutien clair (Parti du progrès et Parti libéral - mouvements politiques d'idéologie fasciste - et Parti de la démocratie chrétienne) ou réservé de certains partis politiques (Parti populaire démocratique [PPD] et Centre démocratique et social [CDS]). Cependant, le 28 septembre, un communiqué de la présidence de la République déclarait l'inconvenance de la marche de la majorité silencieuse et le 29 septembre le Conseil d'État n'a pas accepté la proposition de Spínola de déclarer l'état d'urgence. La tentative de coup d'État a ainsi été arrêtée et le 30 septembre Spínola démissionna. Costa Gomes est alors nommé président de la République et Vasco Gonçalves est nommé pour diriger le $3^{e}$ gouvernement provisoire.

Une nouvelle tentative de renversement politique a encore eu lieu le 11 mars 1975. Le coup Palma Carlos et la tentative de marche de la majorité silencieuse étaient tous les deux partis du pouvoir politique, notamment d'António de Spínola, alors président de la République, président de la JSN et commandant en chef des forces armées. Cette nouvelle tentative de coup d'État a elle aussi été faite par Spínola, mais cette fois-ci de l'extérieur $\mathrm{du}$ pouvoir politique. L'objectif était d'éloigner les militaires révolutionnaires et les communistes du pouvoir politique, lequel serait alors repris par Spínola (qui déclarerait 
l'état d'urgence). Néanmoins le putsch ne réussit pas, Spínola se retira à Madrid, la JSN fut dissoute (et remplacée par le Conseil de la Révolution) et le MFA s'institutionnalisa.

Le 26 mars 1975 a donc été formé un $4^{\mathrm{e}}$ gouvernement provisoire. Aussi dirigé par Vasco Gonçalves, ce gouvernement a organisé les premières occupations de terres (au sud du pays) et a décrété la nationalisation de la banque et des assurances. Cependant, le 10 juillet, les éléments du Parti socialiste (PS) et du Parti populaire démocratique (PPD) l'ont abandonné ; cette attitude, destinée à déstabiliser le nouveau gouvernement, a été très critiquée par le Conseil de la Révolution. Le 8 août a été formé le $5^{\mathrm{e}}$ gouvernement provisoire, toujours dirigé par Vasco Gonçalves, avec la participation du Parti communiste portugais (PCP), du Mouvement démocratique portugais-Coalition démocratique électorale (MDP-CDE), des indépendants et des militaires ${ }^{17}:$ «Le $5^{\mathrm{e}}$ gouvernement provisoire a été le plus révolutionnaire jusqu'à nos jours. » (Vasco Gonçalves, dans Cruzeiro, 2002, p. 221.)

35 Le 13 août, un groupe de neuf militaires ${ }^{18}$, étant en désaccord avec les propositions du $5^{\mathrm{e}}$ gouvernement provisoire, entre en confrontation avec celui-ci en faisant publier un document, le Document des Neuf, qui fut très critiqué par la gauche militaire :

Le document avait, dans mon opinion, un objectif fondamental: mettre fin au processus révolutionnaire, aux transformations économiques, sociales et politiques vers le socialisme. Il propose l'idée d'une troisième voie pour le Portugal et exprime la réflexion de gauche de la petite et de la moyenne bourgeoisie ainsi que ses peurs par rapport à l'ascension de la classe ouvrière, en général, et du Parti communiste portugais, en particulier. (Vasco Gonçalves, dans Cruzeiro, 2002, p. 204-205).

Les divisions grandirent ainsi au sein de l'Armée. Les divergences entre le PCP et le PS se sont également accentuées et le PS (appuyant le Document des Neuf) a exigé la démission du $5^{\mathrm{e}}$ gouvernement provisoire. Le 19 août, Otelo Saraiva de Carvalho (un des instigateurs les plus médiatiques du MFA) a élaboré un plan politique avec le Groupe des Neuf et a rompu avec Vasco Gonçalves. Le $5^{\mathrm{e}}$ gouvernement provisoire s'est ainsi retrouvé isolé et a démissionné le 6 septembre. Le 19 septembre a été formé un nouveau gouvernement provisoire dirigé par le vice-amiral Pinheiro de Azevedo. Les divergences politiques ne cessaient cependant pas de s'accentuer. Le Portugal était divisé et en pleine crise politique et institutionnelle: d'un côté, par la gauche révolutionnaire, politique et militaire, et, de l'autre, par les secteurs les plus conservateurs ainsi que par les défenseurs de l'ancien régime.

Le processus révolutionnaire a donc été le résultat d'une lutte constante entre des factions et des idéologies avec des objectifs très différents.

La contre-révolution a atteint son paroxysme le 25 novembre 1975. Un mouvement composé de militaires et de politiques préparait un coup d'État, dont l'objectif était d'empêcher la constitution d'une "Commune de Lisbonne" (Mota, 1976, p. 94), de rétablir la hiérarchie et la discipline au sein des Forces armées, de dissoudre le MFA (déjà en train de s'auto dissoudre pour cause de divisions et de confrontations internes) et de préparer une éventuelle confrontation militaire avec la gauche militaire et les communistes. La guerre civile semblait, plus que jamais, inévitable.

Quelques chefs et participants de la conspiration militaire, ainsi que des unités militaires et des représentants politiques (dont des éléments du PS, du PPD et du CDS), se sont installés provisoirement à Porto. L'objectif était de déclencher une guerre civile pour que les troupes qui s'étaient installées au nord du pays puissent réagir et dérouter les mouvements civils, populaires et militaires qui éventuellement se formeraient au sud ${ }^{19}$ : 
Si la division du Nord et du Sud du pays s'effectuait, le Royaume-Uni nous soutiendrait politiquement et collaborerait avec le Portugal à travers des appuis spécifiques. Ils nous ont promis de faire arriver à Porto du combustible pour les avions ainsi que des armes. (Mário Soares, dans Avillez, 1996, p. 491.)

Pour justifier un éventuel déclenchement militaire à partir du Nord, les conspirateurs ont alors élaboré un plan dans lequel la descente militaire sur Lisbonne serait une réponse à un prétendu coup militaire que les communistes et autres forces de gauche seraient en train de préparer à Lisbonne.

Néanmoins, le mouvement conspiratif du 25 novembre était constitué par des éléments idéologiquement hétérogènes. Par conséquent, les conspirateurs n'étaient pas tous d'accord avec cette délocalisation vers le Nord qui provoquerait le déclenchement d'une guerre civile :

J'admets qu'il y a eu une connivence entre le PS et Pires Veloso, notamment avec l'idée de fuir vers le Nord, laquelle, de mon point de vue, était complètement absurde et ne pouvait que conduire à la guerre. Après tout ce temps, je n'ai pas de difficultés à admettre que le PS, en particulier Mário Soares, a voulu être, une fois de plus, un grand protagoniste au milieu de tout cela, pour finalement apparaître à la fin comme le grand héros. (Melo Antunes in entretien à Maria Manuela Cruzeiro, Indy, 27-11-1998) ${ }^{20}$.

41 Or, Melo Antunes (un élément du Groupe des Neuf), prétendant éviter une confrontation militaire, est ainsi venu affirmer publiquement, le 26 novembre, que le PCP était un élément politique essentiel pour la construction du socialisme et de la démocratie au Portugal. La guerre civile était ainsi freinée et il n'y a eu aucune invasion du sud du pays par des troupes du nord. Par contre, le mouvement conspiratif a été militairement vainqueur et le processus révolutionnaire freiné. Ainsi, suite aux événements du 25 novembre, le MFA s'est dissous et la hiérarchie militaire a été rétablie.

\section{L'opposition économique à l'ancien régime : construire une nouvelle économie basée sur les propositions socialistes}

De la gauche à la droite, pendant les années 1974 et 1975, les partis politiques inséraient dans leurs programmes politiques la construction du socialisme, ou une alternative économique au capitalisme. Ce caractère socialiste affirmé par les partis évoquait également le climat social de l'époque et a été la substance même de la première Constitution post-dictature.

La première Assemblée constituante a été formée le 2 avril 1976 et était constituée par des députés du PS, du PPD, du PCP, du CDS, du MDP-CDE, de l'UDP ${ }^{21}$ et de la province de Macao. C'est au sein de cette Assemblée politiquement hétérogène que la nouvelle Constitution, sans aucune intervention politique des militaires, a été débattue et approuvée. Cette Loi fondamentale nous révèle donc les aspirations populaires d'alors, incluant des propositions telles que :

L'organisation économique et sociale de la République portugaise est basée sur le développement des relations de production socialistes, à travers l'appropriation collective des principaux moyens de production, des terres et des ressources naturelles ainsi que par l'exercice du pouvoir démocratique des classes travailleuses (II, I, art. 80) ; toutes les nationalisations réalisées après le 25 avril 1974 constituent des conquêtes irréversibles de la classe travailleuse (II, I, art. 83) ; 
la réforme agraire est l'un des outils fondamental pour la construction de la société socialiste (II, IV, art. 96) ; le développement du processus révolutionnaire impose, sur le plan économique, l'appropriation collective des moyens de production (art. 10). portugaise comme une conséquence directe de la crise économique de 1973 (Muñoz, 1997) ni comme une simple rupture avec les anciennes élites (Munck et Leff, 1997). Ainsi, nous défendons que la crise est l'un des éléments mais non pas le seul ni le plus important qui a permis cette transition. Elle ne peut donc pas être envisagée séparément de la lutte des travailleurs ni des mouvements sociaux et politiques. Tous ces facteurs ont donc permis une usure de la dictature, permettant la création d'un environnement favorable au déclenchement du coup d'État militaire du 25 avril 1974 et justifiant le support populaire qui a suivi. En ce sens, nous considérons que le soutien au mouvement des capitaines par des unités des forces armées qui ne faisaient pas partie du MFA ainsi que la mobilisation populaire qui s'est fait sentir dès la première heure de la chute du régime ont, tous les deux, permis la transformation caractérielle d'un simple coup d'État militaire en une révolution militaire et populaire: plus qu'une simple rupture, il y a eu une rupture révolutionnaire. Certes, cette rupture se fait contre les anciennes élites (Munck et Leff, 1997), mais celles-ci ne disparaitront pas complètement du nouvel ordre politique.

Deux aspects apparemment contradictoires caractérisent donc la transition révolutionnaire portugaise : il y a eu une rupture révolutionnaire, toutefois les anciennes élites militaires, même si fragilisées, ont su aussi bien s'adapter au nouvel ordre ainsi qu'y participer politiquement. Beaucoup d'éléments de l'ancien régime ont ainsi continué leur action contre-révolutionnaire, notamment au sein de la JSN, dont la figure principale était le général António de Spínola, premier président de la République post-dictature. La transition a donc été le résultat d'une confrontation constante entre les secteurs les plus progressistes et les secteurs les plus conservateurs. Cette lutte aboutit à un compromis qui a eu lieu suite à la contre-révolution du 25 novembre 1975.

Incluant le caractère révolutionnaire dans le processus turbulent qui a caractérisé la transition de la dictature vers un Portugal démocratique, nous devons donc inclure dans ce processus transitionnel l'action des militaires du MFA (la counterelite, si nous suivons la 
typologie de Munck et Leff) ainsi que les mouvements sociaux et politiques révolutionnaires, les non-élites. Tous les deux ont été des acteurs fondamentaux dans ce processus et ont permis, à certains moments de l'Histoire de cette période, d'inverser la corrélation des forces et de ne pas permettre un retour à l'ancien régime. Leur action et leur lutte ne peuvent donc pas être ignorées dans les choix politiques qui ont été faits lors de la réalisation de la Constitution approuvée en 1976. Certains de ses articles nous révèlent d'ailleurs l'importance sociale et politique de ces mouvements qui non seulement revendiquaient la destruction de l'ancien régime mais aussi la construction d'une nouvelle alternative.

Le choix d'une Constitution où l'économie suit un modèle socialiste ${ }^{24}$ est également le résultat d'une nécessité historique et sociale. Or, la fin de la dictature était également la fin d'un système économique qui n'avait pas su assurer les conditions optimales pour le développement du pays: il fallait donc contrarier et opposer un nouveau système économique ${ }^{25}$ au développement capitaliste mis en place par Salazar et suivi par Marcello Caetano.

L'institutionnalisation d'un nouvel ordre économique et social a toutefois suivi un chemin qui s'est éloigné du modèle socialiste qui a prévalu lors de la transition, notamment en raison des pressions (et interventions) internationales, de la corrélation des forces contradictoires pendant le processus révolutionnaire et des tentatives de renversement $\mathrm{du}$ processus révolutionnaire par les anciennes (et aussi nouvelles) élites pour, finalement, aboutir à un modèle, dans un contexte de guerre froide, suivant les orientations socioéconomiques occidentales. La construction de ce modèle a donc eu comme point de départ la contre-révolution du 25 novembre 1975 ; ce modèle s'est imposé graduellement à travers les options politiques des gouvernements majoritaires depuis 1976 et s'est affirmé politiquement lors de l'entrée du Portugal au sein de la Communauté économique européenne (CEE) en 1986. C'est aussi à partir de ce moment que les articles socialistes présents dans la Constitution de la République ont été remis en cause $^{26}$. Ils ont d'ailleurs été progressivement modifiés, permettant l'institutionnalisation au Portugal d'une économie de marché soumise aux contraintes et aux lois de l'accumulation capitaliste. Les anciennes élites économiques ont alors pu, graduellement, rentrer au Portugal et récupérer leurs positions antérieures. Les anciennes élites sont ainsi redevenues, quelques années après la révolution, les nouvelles élites.

51 Huntington, à l'inverse de ce que nous venons de défendre, affirme que le 25 novembre 1975 a été la consécration de la démocratie au Portugal (1993, p. 5). Cette affirmation contrarie ainsi la thèse de l'avènement d'une contre-révolution. Or, ces différentes visions du même moment politique mettent en valeur les différentes définitions de la notion même de démocratie. Est-elle, comme Huntington le défend, dans la suite des études réalisées par Schumpeter, un simple procédé, excluant, ainsi, le contenu des décisions déterminées et imposées par le pouvoir politique ? Où celle-ci n'est-elle qu'une définition du système d'organisation sociopolitique occidental, dans lequel on nous impose un ordre juridique, économique et politique, à travers lequel on domine les secteurs et les classes adversaires ? La notion même de démocratie nous mène ainsi à la notion de transition démocratique et, par conséquent, à la notion de transition démocratique révolutionnaire. Est-ce que les changements sociopolitiques et économiques opérés lors de la révolution des $F i l l e t s$ ont été une partie d'un processus démocratique révolutionnaire ou la démocratisation ne s'est-elle implantée que lorsqu'un ordre politique et économique a été imposé par une élite politique et militaire? $\mathrm{Ne}$ 
pouvant pas répondre à ces questions dans ce travail, nous les réserverons à une étude postérieure.

\section{BIBLIOGRAPHIE}

AVILLEZ M. J., Soares, Ditadura e Revolução, Lisbonne, Público, 1996.

CARVALHO O. S., Alvorada em Abril, Lisbonne, Bertrand, 1977.

CRUZEIRO M. M., Vasco Gonçalves: um General na Revolução, Lisbonne, Editorial Notícias, 2002.

CUNHAl Álvaro, A Verdade e a Mentira na Revolução de Abril, Lisbonne, Avante!, 1999.

HUNTINGTON Samuel P., The third wave: democratization in the late twentieth century, University of Oklahoma Press, 1993.

мотA J. G., A Resistência. O Verão Quente de 1975, Lisbonne, Expresso, 1976.

MUNCK Gerardo L. et LEFF Carol Skalnik, « Modes of transition and democratization: South America and Easter Europe in Comparative Perspective », Comparative Politics, vol. 29, $\mathrm{n}^{\circ} 3$, avril 1997, p. 343-362.

PALACIOS CEREZALES D., O poder caiu na rua. Crise de Estado e acções colectivas na Revolução Portuguesa, Lisbonne, Imprensa Ciências Sociais, 2003.

MUÑoz R. D., « A crise económica e as transições para a democracia: Espanha e Portugal em perspectiva comparada », Análise Social, vol. XXXII, n 141, 1997, p. 369-401.

OliveIRA MARQues A. H., Breve História de Portugal, Lisbonne, Presença, 2001.

RAMOS DE ALMEIDA P., « Uma década revolucionária em África e em Portugal », 0 Militante, n² 260, septembre-octobre 2002, p. 37-45.

\section{NOTES}

1. Voir G. L. Munck et C. S. Leff, « Modes of Transition and Democratisation. South America and Eastern Europe in Comparative Perspective », p. 343.

2. Voir S. P. Huntington, The third wave: democratization in the late twentieth century, p. 5-15.

3. Ibidem, p. 16.

4. Ibid., p. 17.

5. Police de vigilance et de défense de l'État.

6. Voir S. P. Huntington, The third wave: democratization in the late twentieth century, p. 18.

7. Mouvement populaire pour la libération de l'Angola.

8. Parti africain pour l'indépendance de la Guinée et du Cap-Vert.

9. Mouvement pour la libération de Sao Tomé-et-Principe.

10. Front de libération du Mozambique.

11. Le Parti communiste portugais a été fondé en 1921. 
12. Huntington avait déjà étudié l'aspect économique dans la transition d'un régime autoritaire à un régime démocratique. Pour cet auteur, la croissance économique globale des années 1960 était une des cinq variables qui ont précédé la « troisième vague de démocratisation » (1993, p. 51-59). 13. En 1973 un mouvement des capitaines de l'Armée a exposé au gouvernement des revendications à caractère professionnel. Ce mouvement s'est propagé à d'autres branches des forces armées (forces aériennes et marines) et a élargi ses revendications.

14. Organes provisoires du pouvoir mis en place lors de la Révolution : Commission coordinatrice du programme du MFA (organisme politique et militaire du MFA), JSN, gouvernement provisoire, Conseil d'État (qui était composé par 7 membres de la JSN, 7 membres de la Commission coordinatrice du MFA et 7 membres désignés par le président de la République).

15. Mouvement de Salvation Nationale - mouvement qui résulte d'un compromis entre la hiérarchie militaire et le Mouvement des Forces armées (MFA). La JSN est formée le 26 avril à 1 h 15.

16. Police d'intervention et de défense de l'État - Direction générale de sécurité.

17. Sans la participation ni du Parti socialiste (PS) ni des partis politiques positionnés au centreet à droite (CDS et PPD).

18. Ce groupe de militaires constituait une faction modérée du MFA et s'opposait au document politique du MFA présenté le 8 juillet 1975 : Aliance Peuple-MFA: Pour la construction d'une société socialiste au Portugal.

19. La Constitution de la République était déjà élaborée, mais elle n'était pas encore approuvée.

20. Cité dans Álvaro Cunhal, A verdade e a mentira na Revolução de Abril, p. 225.

21. Union démocratique populaire.

22. Prévoyant la construction du socialisme au Portugal - «La République portugaise est un État démocratique, basée sur la souveraineté populaire, le respect et la garantie des libertés et des droits fondamentaux, et dans le pluralisme de l'expression et de l'organisation politique démocratiques, qui a pour objectif d'assurer la transition vers le socialisme à travers la création de conditions pour l'exercice démocratique du pouvoir par les classes travailleuses » («Principes fondamentaux», art. 2) -, la Constitution de la République portugaise de 1976 a mis en place la nationalisation des secteurs basiques de l'économie - «Éliminer et empêcher la formation des monopoles privés à travers les nationalisations ou d'autres moyens ainsi que réprimer les abus du pouvoir économique et toutes les pratiques lésant l'intérêt général » (partie II, titre IV, art. 96) -, un service publique de santé - «Le droit à la protection de la santé est fait à travers la création d'un service national de santé universel, général et gratuit » (partie I, titre III, art. 64) -, une réforme agraire - « La loi peut déterminer que les expropriations des latifundios et des grands propriétaires et entrepreneurs ou actionnaires peuvent ne pas déboucher sur des indemnisations" (partie II, titre $\mathrm{I}$, art. 82) - et a transformé le système d'éducation considérant l'enseignement basique "universel, obligatoire et gratuit " (partie I, titre III, art. 74). Cependant, malgré une organisation économique (et sociale) basée sur un modèle socialiste que la Constitution prévoyait d'approfondir - «L'organisation économique et sociale de la République portugaise se base sur le développement de relations de production socialistes, à travers l'appropriation collective des principaux moyens de production, des sols et des ressources naturelles, et à travers l'exercice du pouvoir démocratique par les classes travailleuses » (partie II, titre I, art. 80) -, le Portugal n'a pas été un pays socialiste. C'est pour cela que nous parlons d'économie d'inspiration socialiste - « Toutes les nationalisations effectuées après le 25 avril 1974 sont des conquêtes irréversibles de la classe travailleuse » (partie II, titre I, art. 83) - et non de régime économique socialiste. Ainsi, même si un modèle a été suivi - «Dans la phase de transition pour le socialisme, il y aura trois secteurs de propriété des moyens de production, de sols et de ressources naturels » : le secteur publique, le secteur coopératif et le secteur privé (partie II, titre II, art. 89) -, il ne l'a pas été dans son intégralité et n'a pas suffi pour instaurer un socialisme au Portugal ou pour considérer le Portugal comme un pays socialiste. 
23. Notons que nous parlons d'atténuation $d u$ caractère socialiste de certaines lois constitutionnelles graduellement réformées jusqu'à nos jours et non pas d'atténuation du socialisme au Portugal. Ainsi, il y a atténuation du caractère socialiste dans les tentatives de dissolution de la réforme agraire, dans les attaques successives contre le Service national de santé, dans les tentatives de privatisation de l'Éducation nationale, dans le recul des nationalisations des secteurs basiques de l'économie (banques, assurances, construction navale, hôpitaux, terres...).

24. Notons que nous ne considérons pas que l'organisation économique portugaise ait été une économie socialiste mais qu'elle a suivi un modèle socialiste.

25. Ainsi, la Constitution de 1976 prétendait « promouvoir l'égalité entre les citoyens à travers la transformation des structures économiques et sociales » (partie II, titre I, art. 81) et « impulser le développement des relations de production socialistes » (partie II, titre I, art. 81).

26. L'article 2 des "Principes fondamentaux» de la Constitution portugaise qui définissait la «transition vers le socialisme » sera supprimé suite à la révision constitutionnelle de 1982 (la $2^{\mathrm{e}}$ des 7 révisions constitutionnelles que la Constitution subira jusqu'à nos jours).

\section{RÉSUMÉS}

Dans cet article, nous nous proposons d'analyser la transition qui découle de la chute du régime autoritaire le plus long de l'Europe, le 25 avril 1974, au Portugal. Pour cela nous allons considérer la transition portugaise comme un processus de changement qui s'est effectué à travers une rupture politique et économique avec un régime antérieur (Munck et Leff, 1997) qui a permis la rénovation des élites et l'apparition d'un nouveau cadre institutionnel. Cependant, nous n'attribuerons pas le rôle primordial de la transition au mode de transition et au conflit qui en découle entre les élites et les counterelites (Mouvement des Forces armées) mais à l'action des non-élites. Nous considérerons donc que la transition portugaise s'est effectuée par rupture révolutionnaire.

Cet article a ainsi pour objectif d'analyser les raisons qui ont conduit à la chute du régime salazarista-marcelista et le rôle de chacun des principaux protagonistes de la révolution des œillets et $\mathrm{du}$ processus de transition démocratique, pour finalement comprendre la spécificité du processus révolutionnaire portugais.

In this article, we want to analyse the transition that followed the fall of the longest authoritarian regime in Europe, on 25 April 1974, in Portugal. For that we will consider the Portuguese transition as a process of change which took place through a political and economic break with a previous regime (Munck and Leff, 1997) that allowed the renovation of elites and the emerging of a new institutional organization. Though we aknowledge the crucial role of the nonelites so that the transition could happen, we do not attribute that same role to the model of transition and to the conflict that followed between the elites and the counterelites (Movement of Armed Forces). In that sense, we consider that portuguese transition was made through a revolutionary break.

The purpose of this article is the analysis, at first, of the reasons which led to the fall of salazarista-marcelista regime and then, of the role of each of the revolution's main protagonists and the process of democratic transition, in order to understand the specificities of the Portuguese revolutionary process. 
INDEX

Keywords : Colonial War, democracy, fascism, Portuguese Revolution, transition

Mots-clés : démocratie, fascisme, guerre coloniale, révolution des đEillets, transition

\section{AUTEUR}

ANA SALDANHA

Doctorante, Centre de recherche sur l'imaginaire (CRI), Département d'études lusophones de l'université Stendhal-Grenoble 3 